\title{
A report of probable paroxysmal sympathetic hyperactivity syndrome in a patient with necrotizing fasciitis
}

\author{
Sarah Fraser, MD • Daniel H. Ovakim, MD (1)
}

Received: 28 December 2019/Revised: 15 April 2020/Accepted: 17 April 2020/Published online: 5 May 2020

(c) Canadian Anesthesiologists' Society 2020

\section{To the Editor,}

Paroxysmal sympathetic hyperactivity (PSH) is a well described syndrome of increased sympathetic nervous system activity, typically seen following traumatic brain injury, with rare reports in the setting of central nervous system infection, brain malignancy, and anoxic brain injury following cardiac arrest. ${ }^{1-3}$ We wish to share an unusual case following disseminated Group B Streptococcus infection.

A 51-yr-old man with a medical history notable only for hypertension was vacationing in Jamaica when he developed unilateral knee pain and swelling. This prompted his early return to Canada and presentation to the emergency department where he was diagnosed with septic arthritis in his left knee and necrotizing fasciitis in his left leg and right arm. Synovial fluid and blood cultures were positive for Group B Streptococcus. He was admitted to the intensive care unit (ICU) and underwent several surgical debridement procedures to control the infection. His course in the ICU was complicated by septic shock, acute kidney injury requiring continuous renal replacement therapy, Candida albicans fungemia, and prolonged mechanical ventilation. After 12 ICU days, the patient's clinical status had stabilized, his candidemia had cleared,

\footnotetext{
S. Fraser, MD

Department of Medicine, University of British Columbia,

Victoria, BC, Canada

D. H. Ovakim, MD $(\bowtie)$

Department of Emergency \& Critical Care Medicine, Island

Health Authority, Vancouver, BC, Canada

e-mail: daniel.ovakim@viha.ca
}

Department of Medicine, University of British Columbia, Vancouver, BC, Canada he had transitioned to intermittent hemodialysis, and he was successfully extubated. He remained in the ICU because of ongoing hydromorphone and dexmedetomidine infusions and heavy nursing requirements for wound care and dressing changes. Other than a brief $(<30 \mathrm{sec})$ desaturation episode $\left(\mathrm{SpO}_{2}<75 \%\right)$, there was no documented hypoxemia that could have precipitated anoxic brain injury.

On ICU day 14, two days after extubation, and within one hour of an uneventful dressing change, the patient acutely became tachycardic, hypertensive, diaphoretic, hyperthermic, and hypertonic with extensor posturing. This episode resolved with an increase in both the opioid and dexmedetomidine infusions. For several days, he continued to experience at least one similar episode daily, each lasting 20-45 min. A particularly severe episode presented with a respiratory rate of 40 breaths $\cdot \min ^{-1}$, drenching diaphoresis, severe hypertension (systolic $253 \mathrm{mmHg}$ ), tachycardia at 142 beats $\cdot \mathrm{min}^{-1}$, and hyperthermia $\left(\operatorname{Tmax} 38.6^{\circ} \mathrm{C}\right)$. While these episodes typically followed dressing changes, turns, or bathing, several developed without an identifiable trigger and were not preceded by reductions in opioid doses. In anticipation of ongoing high pain management requirements, he was given scheduled oral hydromorphone prior to weaning the opioid infusion. Extensive investigations into the cause of these episodes were undertaken. There was no identified source of infection and he was empirically treated with broad spectrum antibiotics with no improvement. Thyroid function was normal, as were radiological investigations for pulmonary embolism, intraabdominal abscess, and osteomyelitis. There were no physical exam features to suggest meningoencephalitis. There were no contributory findings from brain imaging and an electroencephalogram was reported as "essentially normal" and showed no sign 
FIGURE Paroxysmal sympathetic hyperactivity - assessment measure

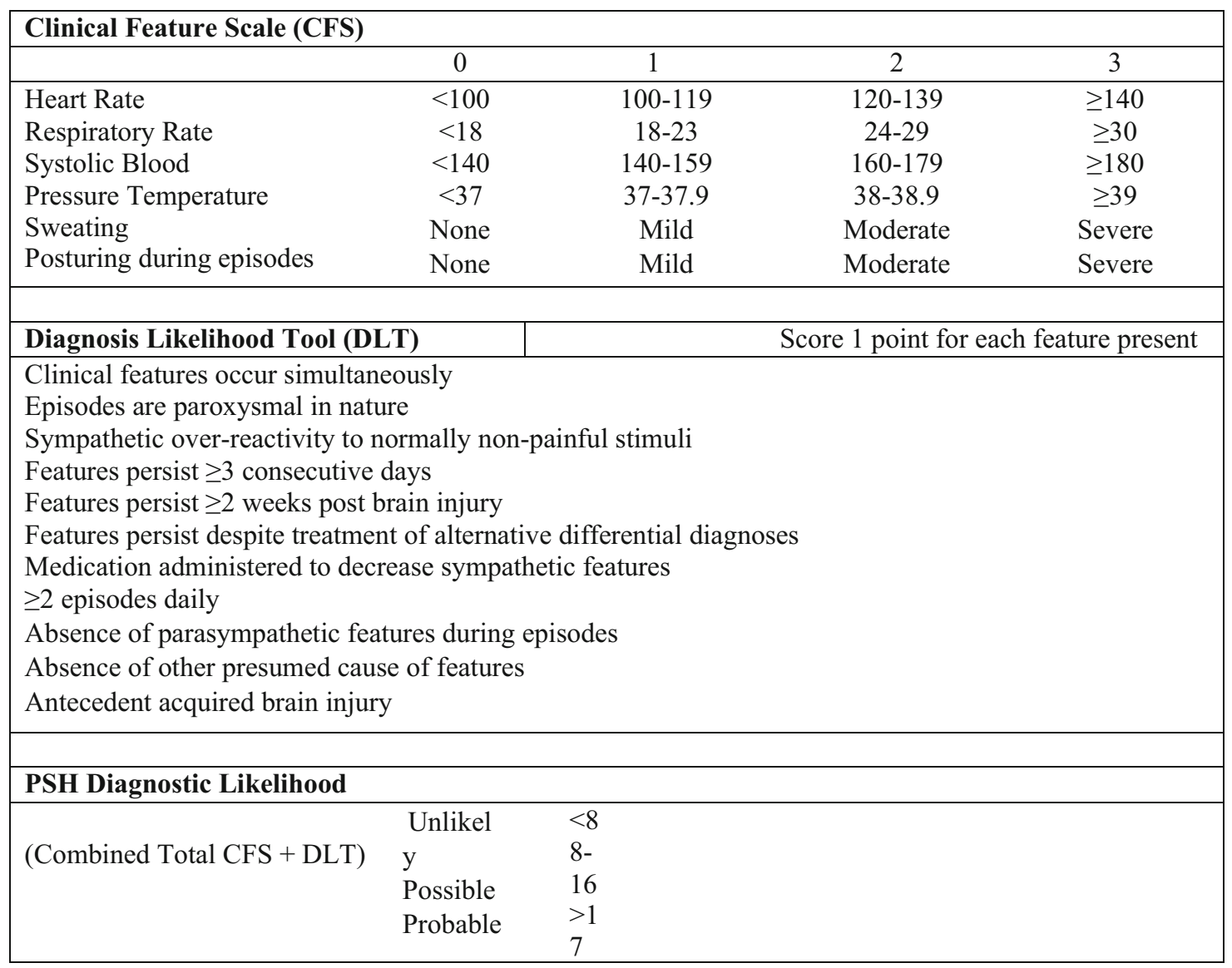

*Adapted from Baguley et al. ${ }^{2}$

FIGURE Adapted from Baguley IJ, Perkes IE, Fernandez-Ortega $J F$, et al. Paroxysmal sympathetic hyperactivity after acquired brain injury: consensus on conceptual definition, nomenclature, and

of epileptiform abnormalities. He was not receiving any antipsychotic medications that could have precipitated neuroleptic malignant syndrome.

Paroxysmal sympathetic hyperactivity was considered, since in both clinical and experimental settings, the majority (approximately 80\%) of paroxysms experienced by patients with PSH occur as allodynic responses to minimal external stimuli such as touching, passive limb movement, bathing, or endotracheal tube suctioning, seemingly consistent with our patient's presentation. ${ }^{4} \mathrm{He}$ was initiated on a regimen of oral propranolol $10 \mathrm{mg}$ three times/day, clonidine $100 \mu \mathrm{g}$ every six hours, and methadone $3 \mathrm{mg}$ three times daily following weaning of the hydromorphone infusion. Following initiation of treatment there were no further episodes during the remainder of his ICU stay, and the patient was successfully transferred from ICU on day 27 for rehabilitation. diagnostic criteria. J Neurotrauma 2014; 31: 1515-20, with permission from Mary Ann Leibert Inc.

Paroxysmal sympathetic hyperactivity most commonly occurs as a complication of traumatic and, less commonly, non-traumatic brain injury and should be considered in patients with features of sympathetic/adrenergic overactivity. The pathophysiology is not fully understood, but the current consensus is that an excitation-inhibition model in which abnormal processing of afferent stimuli within the spinal cord causes normally non-noxious stimuli to overexcite the peripheral sympathetic nervous system. ${ }^{5}$ The PSH-Assessment Measure clinical scoring tool (Figure), a combination of the Diagnosis Likelihood Tool (DLT) addressing the probability of the diagnosis and the Clinical Features Scale (CFS) addressing severity of symptoms, can be used to support a diagnosis (score $>$ 17 , max score 29) or suggest that PSH is unlikely (score $<$ 8). ${ }^{4}$ Our patient scored 21 (DLT 7/11, CFS 14/18) indicating "probable PSH". Many features of PSH resemble opioid withdrawal, which was considered in our 
case; however, our patient was receiving a continuous opioid infusion, and oral hydromorphone (eventually transitioned to oral methadone) was initiated prior to weaning the infusion. There was no recurrence of symptoms when the opioid infusion was discontinued. To our knowledge, there are no documented cases of PSH in non-neurologic illness; however, identification of published cases of PSH is complicated by the extensive use of alternate names. Indeed, Perkes et al. identified 31 synonyms for the condition. ${ }^{1}$ Although there is no goldstandard test for PSH, our patient's "probable" PSHAssessment Measure, the absence of an alternate diagnosis, and a response to treatment suggest a potential case of PSH without brain injury. While clinicians should make every effort to rule out other causes of excessive sympathetic nervous system activity, PSH should be a consideration since delay in recognition and treatment can lead to unnecessary investigations and pharmacotherapy, prolonged hospital and ICU stay, and uncontrolled symptoms that worsen prognosis and increase morbidity.

Funding statement None.

Disclosures None.
Editorial responsibility This submission was handled by Dr. Sangeeta Mehta, Associate Editor, Canadian Journal of Anesthesia.

\section{References}

1. Perkes I, Baguley IJ, Nott MT, Menon DK. A review of paroxysmal sympathetic hyperactivity after acquired brain injury. Ann Neurol 2010; 68: 126-35.

2. Baguley IJ, Perkes IE, Fernandez-Ortega JF, et al. Paroxysmal sympathetic hyperactivity after acquired brain injury: consensus on conceptual definition, nomenclature, and diagnostic criteria. J Neurotrauma 2014; 31: 1515-20.

3. Godoy DA, Panhke P, Guerrero Suarez PD, Murillo-Cabezas F. Paroxysmal sympathetic hyperactivity: an entity to keep in mind. Med Intensiva 2019; 43: 35-43.

4. Meyfroidt G, Baguley IJ, Menon DK. Paroxysmal sympathetic hyperactivity: the storm after acute brain injury. Lancet Neurol 2017; 16: 721-9.

5. Baguley IJ. The excitatory:inhibitory ratio model (EIR model): an integrative explanation of acute autonomic overactivity syndromes. Med Hypotheses 2008; 70: 26-35.

Publisher's Note Springer Nature remains neutral with regard to jurisdictional claims in published maps and institutional affiliations. 